\title{
Intentions to Prevent Weight Gain in Older and Younger Adults; The Importance of Perceived Health and Appearance Consequences
}

\author{
Rebecca J. Beeken ${ }^{\mathrm{a}}$ b Sundus Mahdi ${ }^{\mathrm{b}}$ Fiona Johnson ${ }^{\mathrm{b}}$ \\ Susanne F. Meisel ${ }^{c}$ \\ a Leeds Institute of Health Sciences, University of Leeds, Leeds, UK; ${ }^{b}$ Research Department \\ of Behavioural Science and Health, University College London, London, UK; ${ }^{C}$ Institute of \\ Psychiatry, Psychology \& Neuroscience, King's College London, London, UK
}

\section{Keywords}

Weight gain · Intention $\cdot$ Health $\cdot$ Physical appearance

\begin{abstract}
Objectives: This study investigates whether health and appearance consequences predict intentions to prevent weight gain and whether these relationships differ in younger versus older adults and in men versus women. Methods: UK adults aged 18-26 years (younger adults; $n=584$ ) or $>45$ years (older adults; $n=107$ ) participated in an online survey. Logistic regression assessed associations between intentions to avoid gaining weight and age, gender as well as perceived negative consequences of weight gain for health and appearance. Co-variates were ethnicity, education, weight perception and perceived weight gain vulnerability. Interactions between age, gender and perceived health and appearance consequences of weight gain were also tested. Results: Perceived negative appearance consequences of weight gain predicted weight gain prevention intentions $(O R=9.3, p<0.001)$. Health concerns were not a significant predictor of intentions overall but were a strong predictor for older adults (age $\times$ health concern interaction: $O R=13.6, p>0.01$ ). Conclusion: Concerns about feeling unattractive predict intentions to prevent weight gain. However, health consequences of weight gain are only important motivators for older adults. Future research should identify ways to shift the focus of young people from appearance concerns towards the health benefits of maintaining a healthy weight.

(c) 2018 The Author(s)

Published by S. Karger GmbH, Freiburg
\end{abstract}

Rebecca J Beeken, PhD

Leeds Institute of Health Sciences

University of Leeds

Level 10, Worsley Building, Clarendon Way, Leeds, LS2 9NL, UK

r.beeken@leeds.ac.uk 
Beeken et al.: Intentions to Prevent Weight Gain in Older and Younger Adults; The Importance of Perceived Health and Appearance Consequences

\section{Introduction}

Overweight (BMI $\geq 25 \mathrm{~kg} / \mathrm{m}^{2}$ ) and obesity (BMI $\geq 30 \mathrm{~kg} / \mathrm{m}^{2}$ ) are associated with many non-communicable diseases [1] and together affect more than half of the adult population in the UK [2]. Although effective methods exist to achieve weight loss, sustained weight reduction (>2 years) [3] is difficult to attain [4, 5]. Preventing weight gain in the first instance is an important step to stem the rising tide of obesity [6].

There has been substantial research into the factors that motivate individuals to lose weight, but the literature on motivators for prevention of weight gain is limited. This omission is important since weight loss is recommended only for those who are overweight, but weight gain prevention is also important for those of normal weight, particularly since accumulated environmental pressures and age-related effects result in gradual weight gain over the life course in many people $[7,8]$. An intention to prevent weight gain requires more reflection and anticipation than an intention to lose weight, and it may have different antecedents and determinants and be distributed differently in the population $[9,10]$.

Understanding factors motivating weight gain prevention across groups within a population is a prerequisite for the development of effective, targeted preventative interventions. Although both younger (aged 18-30 years) and older adults (aged $>40$ years) are at risk of weight gain $[8,11-13]$, studies have predominantly focused on young adults $[14,15]$ with less attention given to older people. One cross-sectional study investigated associations of intention to prevent weight gain in a population of young Dutch adults $(n=979)$ [15]. Results indicated that as participants got older they had greater intentions to prevent weight gain, but the age range of the sample was narrow (25-35 years).

Quantitative data on differences in motivation for weight gain prevention between younger and older adults is scarce. Research on motivation to lose weight suggests that older adults may be more strongly motivated by the health risks and medical consequences associated with excess weight, while younger adults' motivation is largely rooted in the desire to improve appearance, partake in social activities and form relationships [16, 17]. A recent qualitative study that interviewed young adult women $(n=25)$ about their use of nutritional food labels suggested that eating healthily to prevent disease was less important during this period of their life than maintaining or improving appearance [18]. Another [19] reported that older women continued to experience concerns associated with appearance but that health concerns were also strong motivators for body weight control in later life.

It might be expected that the intention to prevent weight gain is associated with anticipated consequences of such a gain, but age and gender could moderate the strength of the association. The current study sought i) to establish whether there are differences in the anticipation of the personal health and appearance consequences of weight gain between men and women as well as between older (aged $>45$ years) and younger (aged 18-26 years) adults, ii) to explore whether these concerns predict intentions to prevent weight gain in both sexes and each age group and iii) to examine interactions between age, sex and health and appearance concerns in predicting weight gain prevention intentions. We hypothesised that appearance concerns would predict weight gain prevention intentions to a greater extent in younger adults and women, while health concerns would be stronger predictors in older adults.

\section{Participants and Methods}

\section{Participants and Recruitment}

Participation in the study involved completion of an online questionnaire, hosted through the website SurveyMonkey (www.surveymonkey.com). Participants were recruited from multiple sources: an e-mail invitation was distributed through university mailing lists to all students and staff at University College London 
Beeken et al.: Intentions to Prevent Weight Gain in Older and Younger Adults:

The Importance of Perceived Health and Appearance Consequences

(UCL), and the study was also advertised on social networking sites (Facebook, Twitter and LinkedIn). In addition 'snowball' sampling was used, whereby participants were encouraged to invite friends, family and other contacts to take part. Eligibility criteria were that all participants were resident in the UK and aged either 18-26 years (younger adults) or older than 45 years (older adults). Prospective participants were also excluded if they had a health-related academic or occupational background (defined using the question 'Do you come from a health-related discipline or background (academically or occupationally)', response options: 'yes' and 'no') in order to reduce the risk of participant bias from pro-health attitudes and/or greater knowledge about the health consequences of weight gain. Recruitment advertisements contained a web link that led to the first page of the questionnaire detailing the study aims. Participants were informed that they were free to decide not to participate or to withdraw at any time, and that by progressing onto the next page to begin the survey they were providing consent. At the end of the questionnaire participants had a chance to enter a prize draw.

Of 926 respondents, 237 participants were excluded as they did not meet the eligibility criteria or failed to fully complete the questionnaire. Data was analysed from a total of 689 participants; 582 younger adults and 107 older adults.

\section{Measures}

Demographic variables measured were age, gender, ethnicity and highest level of education completed or under way. For assessment of psychometric and behavioural factors, validated measures were used where available. If fully validated assessment tools were not available, constructs were assessed with measures that had been used in previously published research, and where more than one item was used to measure a construct, inter-item consistency was checked using Cronbach's alpha [20]. Alpha $>0.5$ was regarded as an acceptable cut-off point as has previously been argued in similar cross-sectional studies exploring the predictors of intentions [15]. When constructs met the criteria for internal consistency, the mean item score was computed for analysis. Unless otherwise stated, constructs were assessed on 5-point Likert scales, ranging from strong disagreement to strong agreement.

Weight intentions and perceptions: As in the study by Wammes et al. [15], participants were told that prevention of weight gain should be understood as behaviours such as healthy eating habits and physical activity (e.g. reducing the amount of calories of food in meals and snacks, and activities to increase amount of physical activity), specifically to avoid gaining weight. 'Weight gain' was defined as gaining weight above the amount recommended for the participant's height. The intention to prevent weight gain was measured using a single item, 'Do you intend to try to prevent weight gain?' with response options 'yes' and 'no'. Single item self-report measures are a valid way to measure behavioural intentions [21] and similar measures have been used in other studies of weight gain prevention [15]. A measure of weight perception asked participants to describe their own body weight by selecting one of the following: 'very underweight', 'slightly underweight', 'about right', 'slightly overweight', 'overweight' and 'obese' [22].

Weight gain consequences: Two items ( $\alpha=0.67$ ) were used to assess weight gain risk perception using a comparative personal vulnerability approach. This approach to risk perception was used as it is more strongly associated with health behaviours and intentions than absolute risk estimates [23-25]. First, participants were asked: 'Compared to others of the same sex and age, my chances of gaining weight are ...',with responses recorded on a five-point scale ranging from 'much below average' to 'much above average'. Secondly, participants were asked for agreement with the following: 'If I don't try to prevent weight gain, I would feel very vulnerable to putting on weight'. Responses were recorded on a five-point scale ranging from 'strongly disagree' to 'strongly agree'.

Participants' perceived health consequences in relation to weight gain were assessed with two questions $(\alpha=0.61)$ : 'If I gained weight I would feel ...' (very unhealthy to very healthy), and 'If I gained weight I would feel ...' (very at risk of disease to not at all at risk of disease). All responses were recorded on a fivepoint scale. Mean scores of these two items were calculated as a measure of anticipated health consequences of weight gain. Two items assessed perceived appearance consequences associated with weight gain $(\alpha=$ 0.86): 'If I gained weight I would feel ...' (very dissatisfied with my body to very satisfied with my body; very unattractive to very attractive). As above, mean scores were calculated as a measure of perceived appearance consequences. Health and appearance consequence items were reverse-scored for analysis so that a high score was associated with higher perceived consequences. 
Beeken et al.: Intentions to Prevent Weight Gain in Older and Younger Adults;

The Importance of Perceived Health and Appearance Consequences

Table 1. Frequency or mean and standard deviation (SD) of demographic characteristics and psychosocial constructs

\begin{tabular}{|c|c|c|c|c|c|}
\hline & $\begin{array}{l}\text { Younger adults } \\
(\mathrm{n}=582)\end{array}$ & $\begin{array}{l}\text { Older adults } \\
(\mathrm{n}=107)\end{array}$ & Stat & $\mathrm{p}^{\mathrm{a}}$ & $\begin{array}{l}\text { Total } \\
(n=689)\end{array}$ \\
\hline Age, mean (SD) & $22.0(2.27)$ & $54.9(7.70)$ & $\mathrm{t}=43.8$ & $<0.001$ & $27.1(12.5)$ \\
\hline Gender, $\mathrm{n}(\%$ women $)$ & $441(75.8)$ & $58(54.2)$ & $\chi^{2}=21.1$ & $<0.001$ & $499(72.4)$ \\
\hline Education, $\mathrm{n}(\% \text { post-18 education })^{\mathrm{b}}$ & $571(98.1)$ & $82(76.6)$ & $\chi^{2}=84.2$ & $<0.001$ & $653(94.8)$ \\
\hline Ethnicity, n (\% Caucasian) & $371(63.7)$ & $87(81.3)$ & $\chi^{2}=12.5$ & $<0.001$ & $458(66.5)$ \\
\hline Perceived weight status, n(\% overweight/obese) & $186(32.0)$ & $62(57.9)$ & $\chi^{2}=26.9$ & $<0.001$ & $248(36.0)$ \\
\hline Weight gain prevention intentions, $\mathrm{n}(\%$ yes) & $443(76.1)$ & $92(86.0)$ & $\chi^{2}=5.1$ & 0.024 & $535(77.6)$ \\
\hline Perceived weight gain risk, mean(SD) & $3.09(0.92)$ & $3.26(0.79)$ & $t=2.1$ & 0.040 & $3.11(0.90)$ \\
\hline Anticipated health consequences, mean (SD) & $3.69(0.77)$ & $3.84(0.70)$ & $\mathrm{t}=1.8$ & 0.066 (n.s.) & $3.71(0.76)$ \\
\hline Anticipated appearance consequences, mean (SD) & $4.05(0.82)$ & $3.91(0.74)$ & $\mathrm{t}=1.7$ & 0.092 (n.s.) & $4.03(0.81)$ \\
\hline
\end{tabular}

aSignificance levels calculated using $2 \times 2$ chi-square tests for categorical variables, and t-tests for continuous variables.

bost 18 includes students still studying for a degree that they have not yet completed. The detailed categories reflect highest qualification obtained to date.

Statistical Analyses

Analyses were carried out using the Statistical Package for Social Sciences (SPSS) version 20 (IBM, Armonk, NY, USA). Dichotomous variables were created for ethnicity (white versus non-white) and education (post-18 education versus no post-18 education). The latter considers the issue of asking a predominantly student sample about their highest level of education since by definition it is not finished. Therefore, an 'education beyond 18' variable was created which includes all those who stated that they were students and those with a post-18 qualification. The small sample size within the older age group did not allow us to differentiate more finely between participants of varying educational backgrounds, as frequencies would fall below the $5 \%$ margin.

For perceived weight status, those who perceived themselves as very underweight, slightly underweight and about right were coded as 'not overweight'; the remaining categories were coded as 'overweight' as individual categories were too small for fine-grained comparisons between groups. For five-point scale items, variables were dichotomised for scores $<3$ and scores $\geq 3$. Higher scores indicated high risk of weight gain, high health motivation and high appearance motivation.

Descriptive statistics were completed for all variables. To control for demographic differences between the two age groups, demographic co-variates (gender, ethnicity and education) were included in analyses in cases where they differed between analysis groups. Multivariate analysis of co-variance (MANCOVA) was used to test for main and interaction effects of age group and gender on anticipated health and appearance consequences of weight gain, while controlling for participants' ethnic background, education and perceived weight.

Logistic regression was used to investigate predictors of weight gain prevention intentions. The model included age group, gender, perceived health consequences of weight gain, perceived appearance consequences of weight gain, weight gain risk perceptions, ethnicity and education. A second model tested four two-way interactions: age group by health consequences, age group by appearance consequences, gender by health consequences and gender by appearance consequences.

\section{Results}

The majority of the sample was in the younger age group (85\%), and most were female (72\%) and Caucasian (67\%; table 1). Participants in the younger age group were significantly more likely to be female (76\% vs. 54\%, $\left.\chi^{2}=5.1, \mathrm{p}<0.001\right)$, educated beyond the age of 18 (98\% vs. 77\%, $\left.\chi^{2}=84.2, \mathrm{p}<0.001\right)$ and from an ethnic minority $\left(81 \%\right.$ vs. $64 \%, \chi^{2}=12.5, \mathrm{p}<$ $0.001)$. Just over one-third of the sample (36\%) perceived themselves to be overweight or obese, and this perception was significantly more prevalent in the older age group (58\% vs. $\left.32 \%, \chi^{2}=26.9, \mathrm{p}<0.001\right)$. Around three-quarters of participants $(78 \%)$ stated that they 
Beeken et al.: Intentions to Prevent Weight Gain in Older and Younger Adults: The Importance of Perceived Health and Appearance Consequences

intended to prevent weight gain and this was more common in the older than the younger group ( $86 \%$ vs. $\left.76 \%, \chi^{2}=5.1, p<0.05\right)$.

\section{Anticipated Consequences of Weight gain: Unadjusted Ratings}

Older participants perceived themselves to have a greater risk of weight gain (compared with others of the same age and sex) than younger participants (mean rating $3.3 \mathrm{vs} .3 .1, \mathrm{t}=$ $2.0, p=0.04$ ) (table 1). Men rated themselves to be at lower risk of weight gain than women (mean rating 2.8 vs. $3.2, t=5.2, p<0.001)$. Education post age $18(t=1.7, p=0.09)$ and ethnicity $(t=0.31, p=0.75)$ were not associated with perceived risk of weight gain.

There was no significant difference between the older and younger participants in terms of their concerns about the health consequences $(t=1.8, p=0.07)$ or appearance consequences $(\mathrm{t}=1.7, \mathrm{p}=0.09$ ) of weight gain. Men gave lower ratings than women for both the health (mean rating 3.5 vs. $3.8, t=5.0, p<0.001)$ and appearance consequences $(3.7$ vs. $4.2, t=6.7, p<0.001)$ of weight gain. There was no association between education post age 18 and perceived health consequences of weight gain $(t=0.3, p=0.76)$ or appearance consequences $(t=0.11, p=0.91)$. White ethnic background was not associated with health-related consequences $(t=1.6, p=$ 0.12 ), but white participants gave higher ratings of appearance-related consequences than participants from ethnic minority backgrounds (mean rating 4.1 vs. 3.9, $t=2.1, p=0.04$ ).

\section{Age, Gender and Anticipated Consequences of Weight Gain}

Multivariate analysis of co-variance (MANCOVA) was used to test for main and interactive effects of age group and gender on anticipated health and appearance consequences of weight gain, while controlling for participants' ethnic background, education and perceived weight.

Health consequences of weight gain: There was a main effect of gender on perceived health consequences of weight gain such that female participants gave higher ratings than men (adjusted mean rating 3.vs. 3.56, $F[1,682]=11.2$, $p=0.001$ ). There was a non-significant trend for older participants to perceive more health consequences of weight gain than younger participants (adjusted mean rating 3.77 vs. $3.61, F[1,682]=3.68, p=0.06$ ). No significant interaction between age and gender was found $(F[1,682]=1.80, p=0.18)$.

Appearance consequences of weight gain: There was a strong, significant main effect of gender on ratings of appearance consequences of weight gain, with women giving higher ratings than men (adjusted mean rating 4.04 vs. 3.71, $F[1,682]=16.3, p<0.001$ ) but there was no main effect of age $(F[1,682]=2.1, p=0.15)$. There was a significant interaction between age and gender $(F[1,682]=4.12, p=0.04)$ such that perceived appearance consequences of weight gain decreased in older compared with younger women (adjusted mean 3.89 vs. 4.19) but did not differ in men across the two age groups (adjusted mean 3.72 vs. 3.69).

\section{Intention to Prevent Weight Gain}

The majority of respondents (78\%) intended to prevent weight gain (table 1). Associations between the intention to prevent weight gain and age group, gender as well as perceived consequences of weight gain for health and appearance were examined using logistic regression (table 2). Model 1 examined the effects of age and gender on the intention to prevent weight gain. Co-variates included were education, ethnicity, perceived weight status and perceived vulnerability to weight gain. There was no significant effect of education $(\mathrm{p}=$ $0.835)$ or ethnicity $(p=0.874)$. Individuals who perceived themselves as overweight were more likely to intend prevention than those who saw themselves as not overweight, (OR = 5.98, $\mathrm{p}<0.001$ ). Furthermore, participants who perceived themselves at high risk of weight gain were likely to establish prevention intentions than those who felt at low risk (OR $=18.50$, $\mathrm{p}<0.001)$. Women were more likely than men to report weight gain prevention intentions $(\mathrm{OR}=2.6, \mathrm{p}<0.001)$, but age was not a significant predictor of intentions $(\mathrm{p}=0.118)$. Partic- 
Beeken et al.: Intentions to Prevent Weight Gain in Older and Younger Adults

The Importance of Perceived Health and Appearance Consequences

Table 2. Multivariable logistic regression investigating correlates of weight gain prevention intentions

\begin{tabular}{|c|c|c|c|c|c|c|}
\hline & \multicolumn{3}{|c|}{ Model 1. Main effects } & \multicolumn{3}{|c|}{ Model 2. 2-way interaction effects } \\
\hline & OR & $95 \%$ CI & $\mathrm{p}$ value & OR & $95 \%$ CI & $\mathrm{p}$ value \\
\hline \multicolumn{7}{|l|}{ Education } \\
\hline No post-18 education & 1 & & & 1 & & \\
\hline Post-18 education & 0.85 & $0.19-3.80$ & 0.835 & 0.76 & $0.15-3.84$ & 0.740 \\
\hline \multicolumn{7}{|l|}{ Ethnicity } \\
\hline Non-Caucasian & 1 & & & 1 & & \\
\hline Caucasian & 1.04 & $0.62-1.74$ & 0.874 & 1.03 & $0.61-1.74$ & 0.901 \\
\hline \multicolumn{7}{|l|}{ Gender } \\
\hline Male & 1 & & & 1 & & \\
\hline Female & 2.55 & $1.51-4.29$ & $<0.001$ & 2.84 & $0.88-9.19$ & 0.081 \\
\hline \multicolumn{7}{|l|}{ Age } \\
\hline Younger & 1 & & & 1 & & \\
\hline Older & 2.00 & $0.84-4.79$ & 0.118 & 0.74 & $0.146-3.75$ & 0.716 \\
\hline \multicolumn{7}{|l|}{ Perceived weight status } \\
\hline Not overweight & 1 & & & 1 & & \\
\hline Overweight & 5.98 & $2.42-14.73$ & $<0.001$ & 5.63 & $2.23-14.19$ & $<0.001$ \\
\hline \multicolumn{7}{|l|}{ Perceived weight gain risk } \\
\hline Low & 1 & & & 1 & & \\
\hline High & 18.50 & $7.59-45.09$ & $<0.001$ & 20.98 & $8.30-53.05$ & $<0.001$ \\
\hline \multicolumn{7}{|l|}{ Perceived health consequences } \\
\hline Low & 1 & & & 1 & & \\
\hline High & 1.40 & $0.70-2.49$ & 0.250 & 0.65 & $0.22-1.94$ & 0.441 \\
\hline \multicolumn{7}{|c|}{ Perceived appearance consequences } \\
\hline Low & 1 & & & 1 & & \\
\hline High & 9.27 & $4.62-18.57$ & $<0.001$ & 15.52 & $4.28-56.21$ & $<0.001$ \\
\hline Age $\times$ health consequences & - & - & - & 13.64 & $1.91-97.32$ & 0.009 \\
\hline Age $\times$ appearance consequences & - & - & - & 0.43 & $0.06-3.20$ & 0.412 \\
\hline Sex $\times$ health consequences & - & - & - & 2.11 & $0.59-7.60$ & 0.252 \\
\hline Sex $\times$ appearance consequences & - & - & - & 0.53 & $0.12-2.36$ & 0.403 \\
\hline
\end{tabular}

ipants who perceived more appearance-related consequences of weight gain were more likely to intend weight gain prevention than those not concerned about appearance consequences, $(O R=9.27, p<0.001)$. However, no main effect of perceived health consequences of weight gain on weight gain prevention intentions was found (OR $=1.40, \mathrm{p}=0.316$ ).

A second model was run including four $2 \times 2$ interaction terms: age $x$ health consequences, age $\mathrm{x}$ appearance consequences, sex $\times$ health consequences and sex $\times$ appearance consequences. There was a significant age $\times$ health consequences interaction $(\mathrm{OR}=13.64, \mathrm{p}<$ 0.009). Adjusted predicted probabilities showed that older adults who gave higher ratings of negative health consequences of weight gain were more likely to intend to prevent weight gain compared with older adults who gave lower ratings (adjusted probability of intending to prevent weight gain 0.94 vs. 0.50 ). However, perception of health consequences was not associated with intention to prevent weight gain in younger participants (adjusted probability 0.85 vs. 0.53$)$. The 'age $\times$ appearance' interaction $(p=0.412)$, the sex $\times$ health interaction $(p=0.252)$ and sex $\times$ appearance interaction $(p=0.403)$ were not significant. 
Beeken et al.: Intentions to Prevent Weight Gain in Older and Younger Adults; The Importance of Perceived Health and Appearance Consequences

\section{Discussion}

This study investigated age group and gender differences in the intention to prevent weight gain in British adults. In line with our expectations, we found that perceived consequences of weight gain (adjusted for perceived weight, age and demographic co-variates) differed between men and women in the sample, with women perceiving greater negative health and appearance consequences it they were to gain weight. This was an anticipated finding, likely to reflect differences in weight consciousness between women and men [15, 26-28]. Contrary to our hypothesis, there were no significant differences between the two age groups in terms of the extent to which they perceived negative health and appearance consequences although, among women, older participants reported fewer perceived consequences of weight gain for their appearance compared with younger women.

Across the sample as a whole, the majority of participants (78\%) intended to prevent weight gain. Gender, perceived weight status, perceived risk of weight gain and appearance concern all independently predicted this intention although health concern was not an independent predictor. While some studies have found that age predicts intention to prevent weight gain, we found no association. This may reflect the different age ranges tested in previous studies that have typically focused on narrower and younger age ranges $[15,17]$. It is possible that an association between age and weight gain prevention is non-linear, and future research should establish the profile of intentions across the life course. There was, however, an age $\times$ health concern interaction; older adults who anticipated greater negative health consequences of weight gain were more likely to report an intention to prevent weight gain, whereas anticipated health consequences were not associated with an intention to prevent weight gain in the younger group.

Contrary to our hypothesis, appearance concerns were not a stronger motivator to prevent weight gain in younger compared with older adults. Previous studies have highlighted the importance of looking attractive within young samples [15, 20, 26] but less research has explored appearance concerns within older samples. A recent study on motivations for weight loss demonstrated that older adults place high value on both health and appearance [16], and our findings suggest the same may be the case for weight gain prevention. The fact that appearance concerns were equally motivating for both younger and older adults in our sample may reflect the pervasive association between a lean body weight and attractiveness, which exists irrespective of age within our society and is perpetuated by the emphasis on appearance in the media $[29,30]$.

After perceived risk of weight gain, appearance concern was the strongest predictor of intention to prevent weight gain. While it is encouraging that intentions to prevent weight gain were high in our sample, it is of concern that this potentially reflects the stigmatising view that overweight is unattractive $[31,32]$. Any interventions seeking to increase motivation for weight gain prevention by harnessing this concern could risk exacerbating the negative outcomes associated with a poor body image and could even predispose to greater weight gain [33].

With respect to health concerns, previous studies have shown that younger adults are less motivated by these when it comes to weight loss [16, 17, 34] and food choice [18], and this was also the case in our study on weight gain prevention. Qualitative research has shown that with age, there is an increased awareness of health risks and the need to prevent avoidable diseases [19], which may explain why, in line with our hypothesis, the older adults in our sample were more motivated by health concerns than younger adults.

Given that weight-related illnesses are occurring at younger ages, and taking into account the difficulty of long-term weight loss maintenance $[3,35,36]$, future research should seek to understand how younger people can be engaged with the health consequences of excess 
Beeken et al.: Intentions to Prevent Weight Gain in Older and Younger Adults; The Importance of Perceived Health and Appearance Consequences

weight and to develop interventions that raise awareness of the risks of weight gain without promoting weight stigma. It would be unethical to support or encourage the view that overweight and obesity are less attractive. Furthermore, a focus on appearance could increase the risk of unhealthy weight gain prevention methods. For example, previous research suggests that women may use smoking as a tactic to stay thin out of concern for weight gain as a consequence of cessation [26]. In addition, qualitative research suggests that young female adults can devalue the importance of eating healthily to prevent disease, if they believe that eating less healthily / more restrictively will enhance their attractiveness [18]. Future research should explore how to make early disease prevention more relevant for young adults. For example, immersive reality could be a novel approach that would make the health risks of weight gain more tangible [37].

In line with the previous study on correlates of weight gain prevention in young Dutch adults [15], we also found that perceived risk of weight gain was a strong predictor of intentions to prevent weight gain. Perceived weight status also predicted intentions in our study, although this was not a significant predictor in the study by Wammes et al. [15]. However, this association has been reported elsewhere; Ezendam et al. [38] found that a higher perceived weight status predicted weight gain prevention intentions over a 4-month period in a sample of young adolescents. Similarly, Brug et al [14] demonstrated that self-rated weight status was a stronger correlate of weight maintenance intentions and self-reported actions to avoid weight gain than weight status based on BMI in both adolescents and adults.

Limitations of our study include the method of recruitment; snowball-sampling may to an unknown extent have introduced selection and non-response bias. Consequently, there was an age and gender imbalance in the sample. There were fewer older adults compared with younger adults, and the sample profile was weighted towards women, reflecting the difficulty in engaging men with weight control research [39]. This could prevent the identification of differences between groups, and is in contrast to population estimates for the UK which suggest a roughly equal proportion of men and women living in the UK in 2016 (49\% males and 51\% females) [40]. Unusually our sample was also more ethnically diverse than the UK population; $67 \%$ of our sample were white compared with $87 \%$ of the British population. In addition, the majority of respondents in our sample were educated to degree level or above or were currently studying for a degree, compared with 38\% of the general population in the UK that have HND, degree or higher degree level qualifications or equivalent [40]. Due to the small sample size within the less highly educated group, we were unable to conduct more fine-grained analysis to explore in greater depth any differences by level of education. Therefore, the extent to which the findings from this study can be generalised is limited, as those who are less highly educated and who do not use social media may have different beliefs about the prevention of weight gain.

Furthermore, although we used measures similar to those in previous studies, we aimed to keep the survey as brief as possible to reduce participant burden, which may have led to somewhat crude operationalisations of some variables. However, given the lack of research in this area, the current study provides a suitable starting point for further investigation, although studies with larger, more representative samples are needed before firm conclusions can be made.

The consequences of weight gain measure did not specify the extent of the weight gain, although participants were told at the start of the study that 'weight gain' was defined as gaining weight above the amount recommended for the participant's height. This may have been difficult for participants to interpret which could have affected the reliability of responses. It also did not allow for more detailed analyses around the amount of weight gain required to generate concerns about health and appearance. The amount of weight gain required to cause concern would also likely vary according to perceived weight. Future studies should explore these issues in more depth, potentially using qualitative methodologies. We also explored 
Beeken et al.: Intentions to Prevent Weight Gain in Older and Younger Adults

The Importance of Perceived Health and Appearance Consequences

intentions to prevent weight gain as opposed to behaviour, in line with previous work [15, 38]. However, intentions do not always translate into behaviour change [41], and further studies should incorporate measures of behaviour in order to investigate how the predictors identified in this study relate to actual weight gain prevention.

The results of this study highlight that perceived risk of weight gain and perceived weight status are associated with weight gain prevention intentions. Additionally, females were more likely than males to hold prevention intentions. Results also suggested that both younger and older adults were concerned about the health and appearance consequences of weight gain, but while both age groups were motivated by appearance to prevent weight gain, only older adults were motivated by health concerns. Public health messages that focus on the health risks associated with weight gain may have limited impact with younger audiences, and future research should seek to understand how we can motivate young people to act in response to health concerns and to shift the focus away from appearance consequences of weight gain, which may fuel the stigma of overweight.

\section{Authors' Contributions}

RJB and SFM conceived the study. All authors were involved in study design. SM collected the data, and SM and FJ analysed the data. All authors drafted the manuscript and approved the final version.

\section{Ethical Approval}

The study design and materials were approved by the UCL Research Ethics Committee and the research meets the standards of the Helsinki declaration (1975) and amendments.

\section{Disclosure Statement}

The authors declare that they have no competing interests.

\section{References}

1 Field AE, Coakley EH, Must A, Spadano JL, Laird N, Dietz WH, Rimm E, Colditz GA: Impact of overweight on the risk of developing common chronic diseases during a 10-year period. Arch Intern Med 2001;161:1581-1586.

2 Scantlebury R, Moody A: Health Survey for England 2014: Adult Obesity and Overweight. London, Health Survey For England. 201.,

3 Wing RR, Phelan S: Long-term weight loss maintenance. Am J Clin Nutr 2005;82(1 suppl):222S-225S.

4 Johannsen DL, Knuth ND, Huizenga R, Rood JC, Ravussin E, Hall KD: Metabolic slowing with massive weight loss despite preservation of fat-free mass. J Clin Endocrinol Metab 2012;97:2489-2496.

5 Sumithran P, Prendergast LA, Delbridge E, Purcell K, Shulkes A, Kriketos A, Proietto J: Long-term persistence of hormonal adaptations to weight loss. N Engl J Med 2011;365:1597-1604.

6 World Health Organisation: Obesity: Preventing and Managing the global Epidemic: Report of a WHO Consultation. Geneva, WHO Technical Report Series 894, 2000,

7 Minaker KL: Common clinical sequalae of aging; in Goldman L, Schafer A (eds): Goldman's Cecil Medicine, 24th ed. Philadelphia, Elsevier, 2011.

8 Nooyens AC, Visscher TL, Verschuren WM, Schuit AJ, Boshuizen HC, van Mechelen W, Seidell JC: Age, period and cohort effects on body weight and body mass index in adults: the Doetinchem Cohort Study. Public Health Nutr 2009;12:862-870.

9 Serdula MK, Collins ME, Williamson DF, Anda RF, Pamuk E, Byers TE: Weight control practices of U.S. adolescents and adults. Ann Intern Med 1993;119:667-671.

10 Wardle J, Griffith J, Johnson F, Rapoport L: Intentional weight control and food choice habits in a national representative sample of adults in the UK. Int J Obes Relat Metab Disord 2000;24:534-540.

11 Hankinson AL, Daviglus ML, Bouchard C, Carnethon M, Lewis CE, Schreiner PJ, Liu K, Sidney S: Maintaining a high physical activity level over 20 years and weight gain. JAMA 2010;304:2603-2610. 
Beeken et al.: Intentions to Prevent Weight Gain in Older and Younger Adults

The Importance of Perceived Health and Appearance Consequences

12 Butler SM, Black DR, Blue CL, Gretebeck RJ: Change in diet, physical activity, and body weight in female college freshman. Am J Health Behav 2004;28:24-32.

13 Burke V, Mori TA, Giangiulio N, Gillam HF, Beilin LJ, Houghton S, Cutt HE, Mansour J, Wilson A: An innovative program for changing health behaviours. Asia Pac J Clin Nutr 2002;11(suppl 3):S586-597.

14 Brug J, Wammes B, Kremers S, Giskes K, Oenema A: Underestimation and overestimation of personal weight status: associations with socio-demographic characteristics and weight maintenance intentions. J Hum Nutr Diet 2006;19:253-262.

15 Wammes B, Kremers S, Breedveld B, Brug J: Correlates of motivation to prevent weight gain: a cross sectional survey. Int J Behav Nutr Phys Act 2005;2:1.

16 Robertson A, Mullan B, Todd J: A qualitative exploration of experiences of overweight young and older adults. An application of the integrated behaviour model. Appetite 2014;75:157-164.

17 Gokee LaRose J, Gorin AA, Clarke MM, Wing RR: Beliefs about weight gain among young adults: potential challenges to prevention. Obesity (Silver Spring) 2011;19:1901-1904.

18 Wahlich C, Gardner B, McGowan L: How, when and why do young women use nutrition information on food labels? A qualitative analysis. Psychol Health 2013;28:202-216.

19 Johnston 0, Reilly J, Kremer J: Women's experiences of appearance concern and body control across the lifespan: challenging accepted wisdom. J Health Psychol 2004;9:397-410.

20 Nunnally JC, Bernstein IH, Berge JMT: Psychometric Theory. New York, McGraw-Hill, 1967.

21 Francis J, Eccles M, Johnston M, Walker A, Grimshaw J, Foy R: Constructing questionnaires based on the theory of planned behavior: a manual for health services researchers. Newcastle Upon Tyne, Centre for Health Services Research, 2004. http://openaccess.city.ac.uk/1735/ (last accessed March 2, 2018=

22 Johnson F, Cooke L, Croker H, Wardle J: Changing perceptions of weight in Great Britain: comparison of two population surveys. BMJ 2008;337:a494.

23 Weinstein ND, Kwitel A, McCaul KD, Magnan RE, Gerrard M, Gibbons FX: Risk perceptions: assessment and relationship to influenza vaccination. Health Psychol 2007;26:146-151.

24 Liao Q, Wong WS, Fielding R: Comparison of different risk perception measures in predicting seasonal influenza vaccination among healthy Chinese adults in Hong Kong: a prospective longitudinal study. PLoS One 2013;8:e68019.

25 Dillard AJ, Ferrer RA, Ubel PA, Fagerlin A: Risk perception measures' associations with behavior intentions, affect, and cognition following colon cancer screening messages. Health Psychol 2012;31:106-113.

26 Grogan S, Fry G, Gough B, Conner M: Smoking to stay thin or giving up to save face? Young men and women talk about appearance concerns and smoking. Br J Health Psychol 2009;14:175-186.

27 Brink PJ, Ferguson K: The decision to lose weight. West J Nurs Res 1998;20:84-102.

28 Furnham A, Badmin N, Sneade I: Body image dissatisfaction: gender differences in eating attitudes, selfesteem, and reasons for exercise. J Psychol 2002;136:581-596.

29 Groesz LM, Levine MP, Murnen SK: The effect of experimental presentation of thin media images on body satisfaction: a meta-analytic review. Int J Eat Disord 2002;31:1-16.

30 Grabe S, Ward LM, Hyde JS: The role of the media in body image concerns among women: a meta-analysis of experimental and correlational studies. Psychol Bull 2008;134:460-476.

31 Puhl R, Brownell KD: Bias, discrimination, and obesity. Obes Res 2001;9:788-805.

32 Puhl R, Peterson JL, Luedicke J: Fighting obesity or obese persons? Public perceptions of obesity-related health messages. Int J Obes (Lond) 2013;37:774-782.

33 Puhl R, Suh Y: Health consequences of weight stigma: implications for obesity prevention and treatment. Curr Obes Rep 2015;4:182-190.

34 LaRose JG, Leahey TM, Hill JO, Wing RR: Differences in motivations and weight loss behaviors in young adults and older adults in the National Weight Control Registry. Obesity (Silver Spring) 2013;21:449-453.

35 Must A, Strauss RS: Risks and consequences of childhood and adolescent obesity. Int J Obes Relat Metab Disord 1999;23(suppl 2):S2-11.

36 Dietz WH: Health consequences of obesity in youth: childhood predictors of adult disease. Pediatrics 1998; 101:518-525.

37 Fisher A, Meisel S, Webb T, Fisher B, Cooke C, Newby K: Innerselfie: can we use Immersive Virtual Reality to change cancer risk perceptions and promote physical activity in young people? Front Public Health 2016;86: DOI=10.3389/conf.FPUBH.2016.01.0008. . www.frontiersin.org/10.3389/conf.fpubh.2016.01.00086/event_ abstract (last accessed March 2, 2018).

38 Ezendam NP, Oenema A, Brug J: Predictors of the intention to prevent excessive weight gain in youth. Am J Health Behav 2012;36:472-482.

39 Pagoto SL, Schneider KL, Oleski JL, Luciani JM, Bodenlos JS, Whited MC: Male inclusion in randomized controlled trials of lifestyle weight loss interventions. Obesity (silver Spring) 2012;20:1234-1239.

40 Office for National Statistics: Labour Market Profile - Great Britain. www.nomisweb.co.uk/reports/lmp/ gor/2092957698/report.aspx (last accessed March 2, 2018).

41 Sheeran P, Webb TL: The intention-behaviour gap. Soc Personality Psychol Compass 2016;10:503-518. 